\title{
Sorafenib and nilotinib resensitize tamoxifen resistant breast cancer cells to tamoxifen treatment via estrogen receptor $\alpha$
}

\author{
ASTRID M. PEDERSEN, SUSAN THRANE, ANNE E. LYKKESFELDT and CHRISTINA W. YDE
}

Breast Cancer Group, Cell Death and Metabolism, Danish Cancer Society Research Center, 2100 Copenhagen $\emptyset$, Denmark

Received June 12, 2014; Accepted July 17, 2014

DOI: 10.3892/ijo.2014.2619

\begin{abstract}
Tamoxifen-resistant breast cancer is a major clinical problem and new treatment strategies are highly warranted. In this study, the multitargeting kinase inhibitors sorafenib and nilotinib were investigated as potential new treatment options for tamoxifen-resistant breast cancer. The two compounds inhibited cell growth, reduced expression of total estrogen receptor $\alpha$ (ER), Ser118-phosphorylated ER, FOXA1 and AIB1 and resensitized tamoxifen-resistant cells to tamoxifen. The ER downmodulator fulvestrant exerted strong growth inhibition of tamoxifen-resistant cells and addition of sorafenib and nilotinib could not further suppress growth, showing that sorafenib and nilotinib exerted growth inhibition via ER. In support of this, estradiol prevented sorafenib and nilotinib mediated growth inhibition. These results demonstrate that sorafenib and nilotinib act via ER and ER-associated proteins, indicating that these kinase inhibitors in combination with tamoxifen may be potential new treatments for tamoxifen-resistant breast cancer.
\end{abstract}

\section{Introduction}

Breast cancer is the most common cancer in women in the western world with the majority of tumors being classified as estrogen receptor $\alpha$ (ER) positive. Tamoxifen treatment is the standard first-line therapy for premenopausal women with ER positive breast cancer; however, resistance to treatment is a major clinical problem, and about one third of patients receiving adjuvant treatment and almost all patients with advanced disease develop resistance (1). The mechanisms behind tamoxifen resistance are poorly understood. Tamoxifen is a selective ER modulator that can exert both antagonistic and agonistic effects. In estrogen responsive breast cancer cells, binding of tamoxifen to ER antagonizes the growth

Correspondence to: Dr Christina Westmose Yde, Breast Cancer Group, Cell Death and Metabolism, Danish Cancer Society Research Center, Strandboulevarden 49, 2100 Copenhagen $\varnothing$, Denmark E-mail: cwy@cancer.dk

Key words: breast cancer, tamoxifen resistance, estrogen receptor, receptor tyrosine kinase inhibitors, cell signaling stimulation from estrogen (2). We have previously shown that tamoxifen-resistant cell lines rely on ER for growth and that tamoxifen acts as an agonist (3). ER co-regulators play important roles for determining whether tamoxifen functions as an antagonist or agonist (4). Activated ER is associated to the chromatin through actions of pioneer factors, e.g., the forkhead protein FOXA1, which ensures the opening of the chromatin allowing binding of other proteins to the DNA (5). When estrogen binds to ER, the AF2 domain of the receptor is activated, leading to binding of co-activators and initiation of transcription. One such co-activator is amplified in breast cancer 1 (AIB1), which is a member of the p160 steroid receptor co-activator family (6). AIB1 has been shown to be phosphorylated by mitogen activated protein kinase (MAPK), thereby enhancing its transactivation potential (7). Other co-activators can bind to the AF1 domain of ER, mediated by ligand-independent activation of the receptor (8).

Ligand-independent activation of ER can occur through phosphorylation by receptor tyrosine kinase (RTK) pathways $(9,10)$. RTKs are high affinity transmembrane receptors for many growth factors, hormones and cytokines. Activation of RTKs leads to the activation of different signaling pathways, including the MAPK and phosphoinositide-3-kinase (PI3K) pathways, which play important roles in cell growth (11). Therefore, treatment with kinase inhibitors may be a way to target tumor growth and prevent the ligand-independent activation of ER. Sorafenib is an inhibitor of vascular endothelial growth factor receptor (VEGFR), platelet derived grow th factor receptor (PDGFR), rearranged during transformation (RET) and Raf (12), where especially the kinase RET has been associated with tamoxifen resistance (13). Sorafenib is approved for treatment of advanced kidney and liver cancer $(14,15)$ and is currently in clinical trials for treatment of advanced breast cancer $(16,17)$. Studies in mice have shown that treatment with sorafenib inhibits breast tumor growth and angiogenesis (18). Nilotinib is an inhibitor of PDGFR, c-Kit and the fusion protein $\mathrm{Bcr} / \mathrm{Abl}$, and was designed as a high affinity molecule against $\mathrm{Bcr} / \mathrm{Abl}$ to treat chronic myeloid leukemia (CML) after resistance to imatinib $(19,20)$. Nilotinib is now used as front line treatment for newly diagnosed CML patients (21). Preclinical studies show that nilotinib has a growth inhibitory effect on long-term estrogen deprived (LTED) MCF-7 breast cancer cells via ER (22).

In this study, we tested sorafenib and nilotinib as potential new treatments for tamoxifen-resistant breast cancer. We 
show that the compounds preferentially inhibit the growth of tamoxifen-resistant cells compared with parental MCF-7 cells and resensitize the resistant cells to tamoxifen. We also show that sorafenib and nilotinib downregulate total and phosphorylated ER as well as FOXA1 and AIB1, and that sorafenib and nilotinib have no effect in presence of estradiol supporting a mechanism of action via ER.

\section{Materials and methods}

Cell lines and culture conditions. The MCF-7 cell line was originally obtained from the Human Cell Culture Bank (Mason Research Institute, Rockville, MD, USA) and adapted to grow in medium with low estrogen (MCF-7/S0.5; hereafter called MCF-7) (23). The tamoxifen resistant cell lines; MCF-7/TAM ${ }^{\mathrm{R}}-1$, MCF-7/TAM ${ }^{\mathrm{R}}$-4, MCF-7/TAM ${ }^{\mathrm{R}}-7$ and $\mathrm{MCF}^{-7 / \mathrm{TAM}^{\mathrm{R}}-8}$ (hereafter called $\mathrm{TAM}^{\mathrm{R}}-1, \mathrm{TAM}^{\mathrm{R}}-4, \mathrm{TAM}^{\mathrm{R}}-7$, and $\mathrm{TAM}^{\mathrm{R}}-8$, respectively) were established from the $\mathrm{MCF}-7$ cell line as previously described $(3,24)$. All cell lines were maintained at $37^{\circ} \mathrm{C}$ in humidified air with $5 \% \mathrm{CO}_{2}$ in phenol-red-free DMEM/F12 medium (Invitrogen, Carlsbad, CA, USA) containing 1\% FBS (Invitrogen), $2.5 \mathrm{mM}$ L-glutamax (Invitrogen) and $6 \mathrm{ng} / \mathrm{ml}$ insulin (Sigma-Aldrich, St. Louis, MO, USA). Growth medium for tamoxifen-resistant cell lines was supplemented with $1 \mu \mathrm{M}$ tamoxifen (Sigma-Aldrich). The MCF-7 cell line used in this study was authenticated in January, 2014 by DNA profiling using short tandem repeat loci performed by Leibniz-Institut DSMZ (Braunschweig, Germany) and found to be matching the genetic profile reported for the MCF-7 cell line (DSMZ ACC 115).

Growth experiments. For tamoxifen dose-response experiments, and the experiments where cells were grown without tamoxifen, tamoxifen was withdrawn from the growth medium of resistant cells one week prior to treatment. For dose-response experiments with inhibitors, cells were grown in standard growth medium, i.e., containing $1 \mu \mathrm{M}$ tamoxifen for resistant cell lines. Cells were seeded two days prior to treatment with the indicated concentrations of tamoxifen (Sigma-Aldrich), 4-OH tamoxifen (Sigma-Aldrich), fulvestrant (ICI182,780; Tocris Bioscience, Bristol, UK), estradiol (Sigma-Aldrich), sorafenib (Selleck, Houston, TX, USA) or nilotinib (Selleck). Control cells received similar amount of vehicle as the treated cultures; i.e., $0.1 \%$ ethanol or $0.1 \%$ DMSO. Treatment medium was renewed after three days and cell number was determined after 5 days of treatment using a crystal violet stain colorimetric assay as previously described (25). All growth experiments were performed with triplicate samples or more and repeated at least twice with similar results.

Western blot analysis. MCF-7, $\mathrm{TAM}^{\mathrm{R}}-1$ and $\mathrm{TAM}^{\mathrm{R}}-4$ cells were seeded in their standard medium and treated for 1-72 $\mathrm{h}$ with the indicated compounds. Cells were harvested in RIPA buffer and western blot analysis was performed as previously described (26). Immunostaining was performed overnight with primary antibodies directed against the following proteins: Akt (9272), phospho-Ser473-Akt (9271), phospho-Ser118-ER $\alpha$ (2511), ERK1/2 (9102) and phospho-Thr202/Tyr204-ERK1/2 (4377) from Cell Signaling Technology (Danvers, MA, USA); ER $\alpha$ (RM-9101), Hsp70 (MS-482-PO) from Neomarkers (Fremont, CA, USA); $\beta$-actin (A5441) from Sigma-Aldrich; PARP (511024) and AIB1 (611105) from BD Biosciences (San Jose, CA, USA) and FOXA1 (23738) from Abcam (Cambridge, MA, USA). Western blot analyses were done on at least two independent sets of lysates with similar results.

LDH assay. MCF-7 and TAM ${ }^{\mathrm{R}}-4$ cells were seeded in 96-well plates and treated with the indicated concentrations of sorafenib or nilotinib. To investigate inhibition of cell death, cells were treated with $5 \mu \mathrm{M}$ z-Val-Ala-dl-Asp-fluoromethylketone (zVAD-fmk) (Bachem, Torrance, CA, USA) or $85 \mu \mathrm{M}$ z-Phe-Ala-fluoromethylketone (zFA-fmk) (Calbiochem, Darmstadt, Germany). Cytotoxicity was measured using lactate dehydrogenase (LDH) cytotoxicity assay (Roche, Basel, Switzerland) according to the manufacturer's instructions. All experiments were repeated at least twice with similar results.

Statistical analysis. Statistical analyses were performed on results from cell growth assays and LDH assays in order to determine significant differences between groups. Data from the representative experiments are shown and expressed as mean \pm SD as a percentage of controls or as cytotoxicity values $\pm \mathrm{SD}$. Group comparisons were done using a two-tailed t-test with Bonferroni adjusted p-values for multiple testing. The level of statistical significance was set to $\mathrm{p}<0.05$ and indicated by asterisks in the figures.

\section{Results}

Treatment with sorafenib or nilotinib preferentially inhibits the growth of the tamoxifen-resistant cell lines compared with MCF-7. The effect of sorafenib and nilotinib on tamoxifen-resistant cell growth was explored using four different tamoxifen-resistant breast cancer cell lines, derived by long-term treatment of MCF-7 cells with tamoxifen. Both the parental MCF-7 cell line and the four tamoxifen-resistant cell lines were growth inhibited in a dose-dependent manner by treatment for 5 days with sorafenib or nilotinib (Fig. 1). The tamoxifen-resistant cell lines $\mathrm{TAM}^{\mathrm{R}}-4$, $\mathrm{TAM}^{\mathrm{R}}-7$ and $\mathrm{TAM}^{\mathrm{R}}-8$ were significantly more growth inhibited by treatment with $0.25,0.5,0.75$ and $1 \mu \mathrm{M}$ sorafenib when compared with MCF-7 cells (Fig. 1A). Cell growth of TAM ${ }^{\mathrm{R}}-1$ cells was significantly more reduced using 0.5 and $0.75 \mu \mathrm{M}$ sorafenib compared with MCF-7 cells (Fig. 1A). Preferential growth inhibition was achieved with 0.25 and $0.5 \mu \mathrm{M}$ nilotinib in $\mathrm{TAM}^{\mathrm{R}}-1, \mathrm{TAM}^{\mathrm{R}}-7$ and $\mathrm{TAM}^{\mathrm{R}}-8$, and with $1 \mu \mathrm{M}$ nilotinib, the growth of all four tamoxifen-resistant cell lines was significantly more inhibited compared with MCF-7 (Fig. 1B).

Treatment with sorafenib or nilotinib renders the resistant cells sensitive to tamoxifen inhibition. Next, we investigated whether sorafenib and nilotinib rendered the tamoxifen-resistant cell lines sensitive to tamoxifen treatment. Growth assays showed that the tamoxifen-sensitive, parental MCF-7 cells, grown without sorafenib or nilotinib, were growth inhibited in a dosedependent manner by 5 days of treatment with 4-OH-tamoxifen (Fig. 2A). Adding $0.5 \mu \mathrm{M}$ sorafenib or nilotinib had only modest effect on the 4-OH-tamoxifen response in MCF-7 cells (Fig. 2A). As expected, $\mathrm{TAM}^{\mathrm{R}}-1$ and $\mathrm{TAM}^{\mathrm{R}}-4$ cells, grown without sorafenib or nilotinib, were resistant to 4-OH-tamoxifen treatment and a small agonistic effect was observed (Fig. 2B and C), 

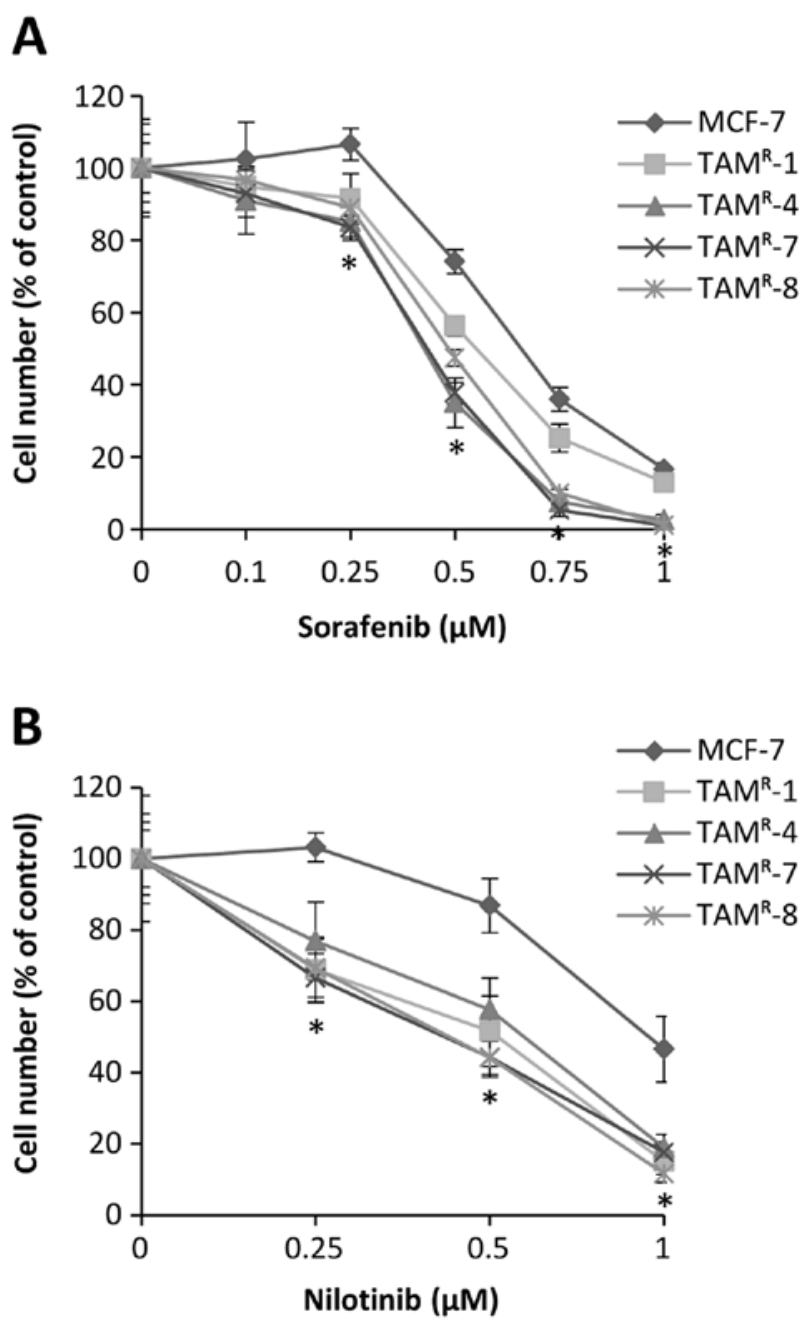

Figure 1. Effect of sorafenib and nilotinib on cell growth. The parental cell line MCF-7 and the four resistant cell lines TAM ${ }^{\mathrm{R}}-1, \mathrm{TAM}^{\mathrm{R}}-4, \mathrm{TAM}^{\mathrm{R}}-7$ and $\mathrm{TAM}^{\mathrm{R}}-8$ were seeded in their standard growth medium and treated with increasing concentrations of (A) sorafenib or (B) nilotinib. Cell number was determined by a crystal violet staining method. The results are expressed relative to the control $(0.1 \%$ DMSO treated). Error bars represent standard deviation (SD) of the mean of at least three replicate values. Asterisks $(*)$ indicate significant difference from MCF-7 cells $(\mathrm{p}<0.05)$.

in agreement with previous findings (3). However, when grown in the presence of $0.5 \mu \mathrm{M}$ sorafenib or nilotinib, which resulted in about $20 \%$ growth inhibition, addition of 4-OH-tamoxifen exerted a further dose-dependent growth inhibition (Fig. 2B and C). The combined treatment inhibited growth of the resistant cell lines to almost the same level as tamoxifen treated MCF-7 cells, demonstrating that sorafenib and nilotinib resensitize the resistant cell lines to tamoxifen treatment. Furthermore, $\mathrm{TAM}^{\mathrm{R}}-4$ cells could not be propagated continuously in presence of $1 \mu \mathrm{M}$ tamoxifen plus $0.5 \mu \mathrm{M}$ sorafenib or nilotinib (data not shown), suggesting that the combined treatment effectively prevents resistant cell growth.

Sorafenib and nilotinib induce ERK/Akt phosphorylation and downregulate total and S118-phosphorylated ER. The effect of sorafenib and nilotinib treatment on downstream signaling was analyzed in MCF-7 and the two tamoxifen-resistant cell lines TAM ${ }^{\mathrm{R}}-1$ and $\mathrm{TAM}^{\mathrm{R}}-4$ (Fig. 3). The cells were short-term
(1-1.5 h) and long-term (24 h) treated with $1 \mu \mathrm{M}$ sorafenib or $1 \mu \mathrm{M}$ nilotinib. Since sorafenib and nilotinib are inhibitors of several RTKs, a decreased activity of MAPK and PI3K pathways would be expected. However, both in MCF-7 and tamoxifen-resistant cell lines, phosphorylation of ERK and Akt was increased upon treatment with sorafenib and nilotinib (Fig. 3A and B). The increase of phosphorylated ERK was observed after only 15 min using concentrations of sorafenib down to $0.25 \mu \mathrm{M}$ in $\mathrm{TAM}^{\mathrm{R}}-4$ cells (Fig. 3C) and a decline in the level of phosphorylated ERK was seen in most experiments after $24 \mathrm{~h}$. Furthermore, in our cell model, we were unable to detect any decrease in phosphorylation of the primary targets RET and PDGFR (data not shown), indicating that sorafenib and nilotinib may act through other targets. We have previously shown that the tamoxifen-resistant cell lines rely on ER for growth (3). ER has several phosphorylation sites, the most well studied being serine 118 (S118) which is an ERK phosphorylation site (27) associated with tamoxifen resistance (28). Interestingly, the levels of both S118-phosphorylated and total ER were decreased upon treatment with sorafenib or nilotinib in the resistant cell lines but not in MCF-7 cells (Fig. 3A and B). Treatment with nilotinib caused a lower level of phosphorylated ER already after $1 \mathrm{~h}$ of treatment. After $24 \mathrm{~h}$ of treatment with sorafenib or nilotinib, ER was clearly reduced in both tamoxifen-resistant cell lines, indicating that sorafenib and nilotinib may act through similar mechanisms, involving reduction of phosphorylated ER and of total ER protein.

Sorafenib and nilotinib downregulate ER independent of tamoxifen. The observed resensitization of the resistant cell lines to tamoxifen (Fig. 2) and the decreased level of ER seen upon treatment with sorafenib and nilotinib (Fig. 3) could indicate that ER plays a role for the effect of the two kinase inhibitors in tamoxifen-resistant cells. We and others have previously shown that treatment with tamoxifen causes an increase in the level of phosphorylated ER, presumably due to a ligand-dependent mechanism caused by tamoxifen $(3,29)$. This tamoxifen-induced increase in S118-phosphorylated ER is seen in Fig. 4A and B, where stabilization of the ER protein, mediated by tamoxifen (30), is also evident. In this experiment, $6 \mathrm{~h}$ of treatment with the kinase inhibitors was included to investigate an intermediate time point between 1 and $24 \mathrm{~h}$. In presence of tamoxifen, sorafenib and nilotinib caused a decrease in S118-phosphorylation of ER after both 6 and $24 \mathrm{~h}$ of treatment (Fig. 4A and B), similar to the results shown in Fig. 3 after $24 \mathrm{~h}$ of treatment. The level of total ER protein was reduced upon 6 and $24 \mathrm{~h}$ of treatment with sorafenib or nilotinib, both in the presence and absence of tamoxifen (Fig. 4A and B), indicating that the sorafenib- and nilotinib-induced ER downregulation occurs independently of tamoxifen. To further investigate the effect of the kinase inhibitors on ER and ER co-regulators, $\mathrm{TAM}^{\mathrm{R}}-4$ cells were treated for $24 \mathrm{~h}$ with increasing concentrations of sorafenib or $1 \mu \mathrm{M}$ sorafenib with and without tamoxifen. A reduction in ER protein was observed with 0.5 and $1 \mu \mathrm{M}$ sorafenib (Fig. 4C). The levels of ER pioneer factor FOXA1 and the co-activator AIB1 were also decreased upon treatment with $1 \mu \mathrm{M}$ sorafenib, though the decrease in AIB1 protein level was less than for ER and FOXA1. In TAM ${ }^{\mathrm{R}}-4$ cells treated with $1 \mu \mathrm{M}$ sorafenib with or without tamoxifen, FOXA1 was decreased by sorafenib in a tamoxifen indepen- 
A

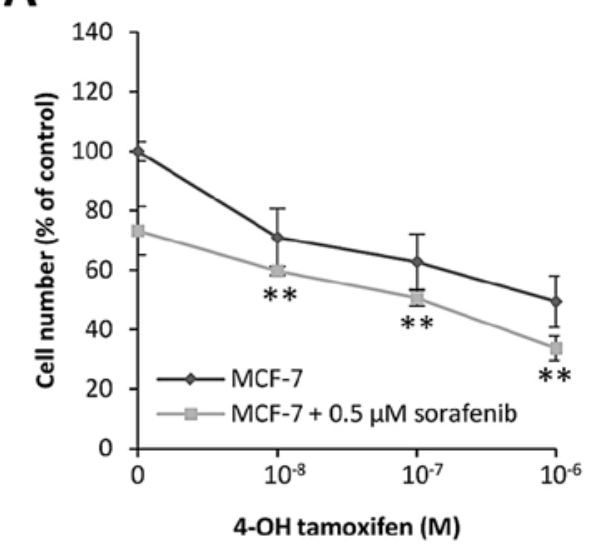

B

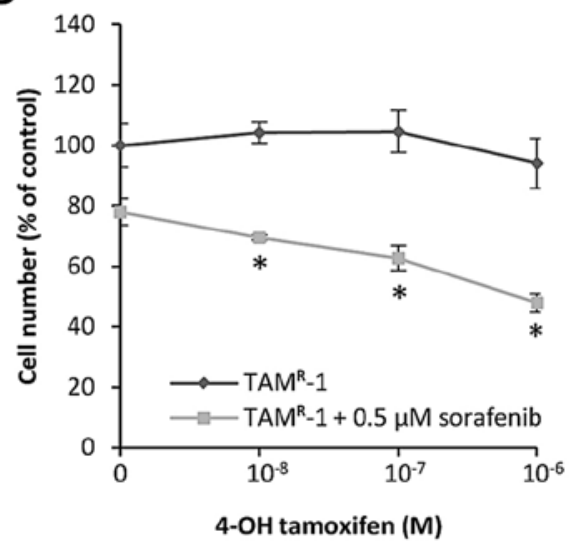

C

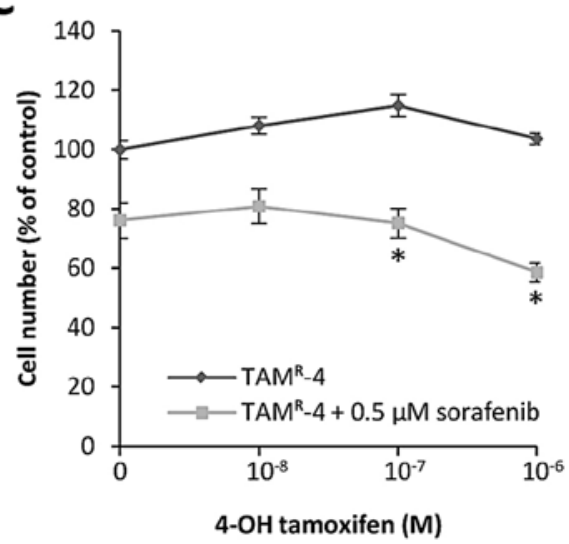

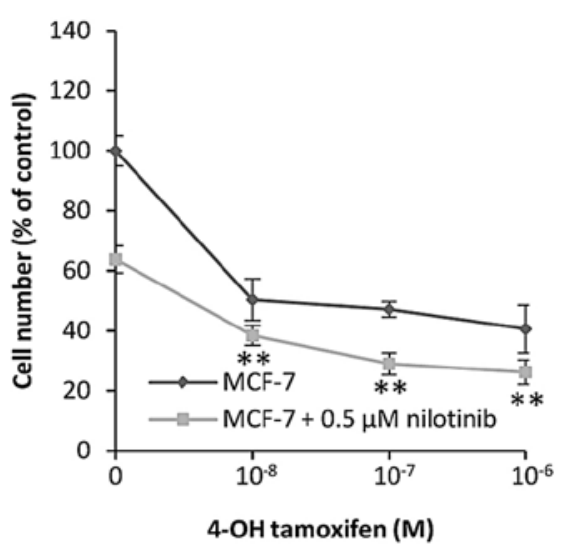
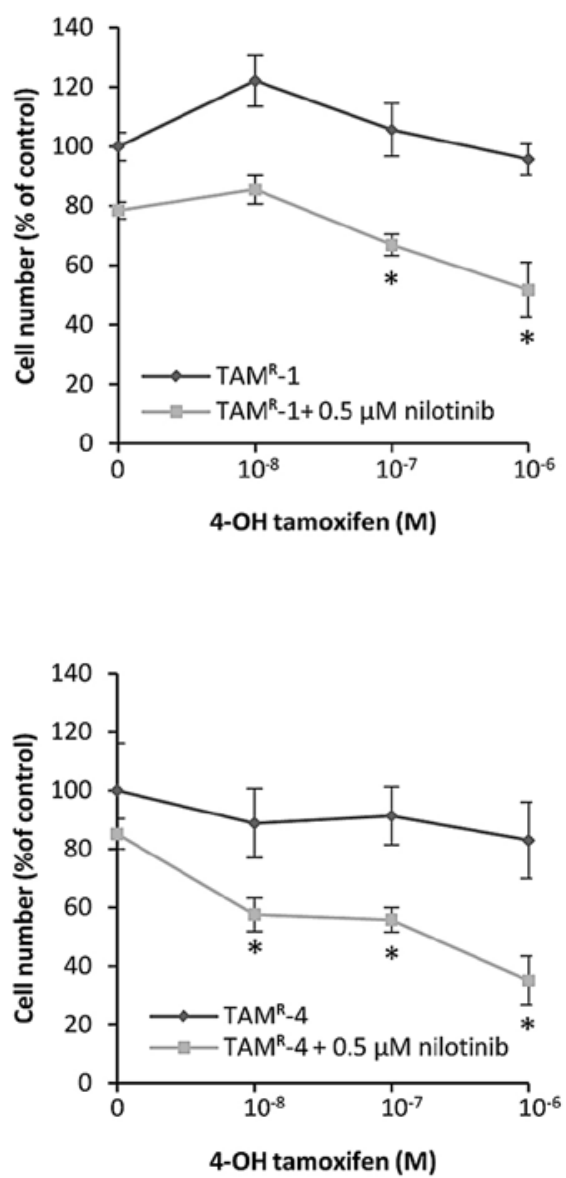

Figure 2. Effects of combined treatment with tamoxifen and sorafenib or nilotinib on cell growth. One week before seeding, tamoxifen was withdrawn from the medium of the resistant cell lines. (A) MCF-7, (B) TAM ${ }^{\mathrm{R}}-1$ and (C) TAM ${ }^{\mathrm{R}}-4$ cells were treated with increasing concentrations of 4-OH-tamoxifen with or without $0.5 \mu \mathrm{M}$ sorafenib or $0.5 \mu \mathrm{M}$ nilotinib. Cell number was determined by a crystal violet staining method. The results are expressed relative to the control $(0.1 \%$ EtOH treated). Error bars represent SD of the mean of at least three replicate values. Asterisks (*) indicate significant growth inhibition compared with cells grown without tamoxifen $(\mathrm{p}<0.05)$.

dent manner (Fig. 4C). Concerning AIB1, it seemed that the decrease in AIB1 protein after sorafenib treatment was more pronounced in the presence than in the absence of tamoxifen (Fig. 4C). A substantial decrease in the level of FOXA1 and a minor reduction in AIB1 were also observed in $\mathrm{TAM}^{\mathrm{R}}-4$ cells upon $24 \mathrm{~h}$ of treatment with 0.5 and $1 \mu \mathrm{M}$ nilotinib (Fig. 4D).
ER is required for the growth inhibiting effect of sorafenib and nilotinib. To further study the importance of ER for inhibition of tamoxifen-resistant cell growth by sorafenib and nilotinib, $\mathrm{TAM}^{\mathrm{R}}-4$ cells were treated with fulvestrant or estradiol (E2) in combination with the kinase inhibitors (Fig. 5). Fulvestrant binds competitively to ER resulting in degradation of the 

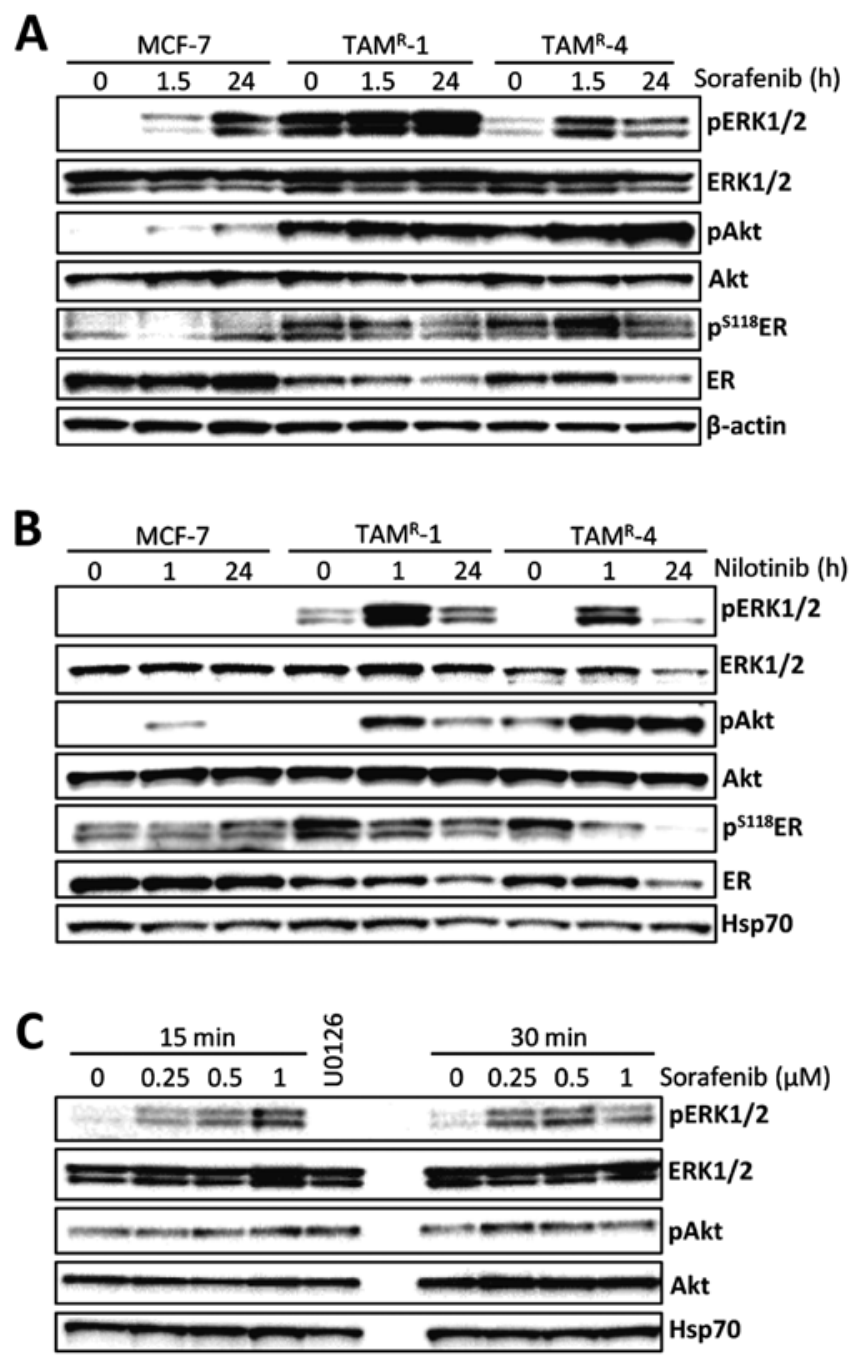

Figure 3. Effect of sorafenib and nilotinib on ERK, Akt and ER expression and phosphorylation. MCF-7, TAM ${ }^{\mathrm{R}}-1$ and $\mathrm{TAM}^{\mathrm{R}}-4$ cells were grown in their standard growth medium. DMSO $(0.1 \%)$ was used as control. Heat shock protein 70 (Hsp70) and $\beta$-actin were used as loading controls. (A) The cells were treated for 1.5 or $24 \mathrm{~h}$ with $1 \mu \mathrm{M}$ sorafenib before harvest for western blot analysis (B) The cells were treated for 1 or $24 \mathrm{~h}$ with $1 \mu \mathrm{M}$ nilotinib before harvest. (C) $\mathrm{TAM}^{\mathrm{R}}-4$ cells were treated for 15 or 30 min with increasing concentration of sorafenib as indicated before harvest for western blot analysis. The selective MEK inhibitor U0126 was used at $5 \mu \mathrm{M}$ concentration for $15 \mathrm{~min}$.

receptor (31). As previously demonstrated (24), fulvestrant inhibited growth of the tamoxifen-resistant cell line $\mathrm{TAM}^{\mathrm{R}}-4$ (Fig. 5A and B). Addition of $0.25 \mu \mathrm{M}$ sorafenib (Fig. 5A) or nilotinib (Fig. 5B) to fulvestrant did not result in further growth inhibition, whereas $0.5 \mu \mathrm{M}$ sorafenib or nilotinib in combination with fulvestrant exerted significantly more growth inhibition than fulvestrant alone. Noteworthy, in combination with estradiol neither $0.25 \mu \mathrm{M}$ sorafenib nor $0.25 \mu \mathrm{M}$ nilotinib affected cell growth (Fig. 5C). Together, these results demonstrate that at low concentrations the growth inhibiting effect of the two kinase inhibitors involves ER. At higher kinase inhibitor concentrations, ER-independent mechanisms may also be involved in the growth inhibitory effect.

High concentrations of sorafenib induce caspase and cathepsin-mediated cell death. To investigate if the observed
ER-independent growth inhibition at higher drug concentrations could be due to cell death, an LDH assay was performed on MCF-7 and TAM ${ }^{\mathrm{R}}-4$ after treatment with 1 or $2 \mu \mathrm{M}$ sorafenib for $72 \mathrm{~h}$. Both cell lines showed increased cell death with increasing sorafenib concentrations and the cell death was significantly higher in $\mathrm{TAM}^{\mathrm{R}}-4$ when compared to MCF-7 cells (Fig. 6A). The broad-range caspase inhibitor z-Val-Ala-dl-Asp-fluoromethylketone (zVAD-fmk) was used to investigate if the sorafenib-induced cell death was due to caspase-mediated apoptosis and the cathepsin inhibitor z-Phe-Ala-fluoromethylketone (zFA-fmk) was used to investigate if the sorafenib-induced cell death was mediated through cathepsins, indicative of cell death by lysosomal membrane permeabilization (32). MCF-7 and TAM ${ }^{\mathrm{R}}-4$ cells were treated with 1 or $2 \mu \mathrm{M}$ sorafenib in combination with zVAD-fmk or zFA-fmk. Cell death was induced by $2 \mu \mathrm{M}$ sorafenib in MCF-7 and could not be significantly prevented by the two inhibitors (Fig. 6B). For TAM ${ }^{\mathrm{R}}-4$ cells, cell death was induced at $1 \mu \mathrm{M}$. However, the cell death could not be inhibited until cytotoxicity was reached with $2 \mu \mathrm{M}$ sorafenib. At this point the cell death of TAM ${ }^{\mathrm{R}}-4$ cells could be inhibited by both the caspase inhibitor zVAD-fmk and the cathepsin inhibitor zFA-fmk (Fig. 6B). Furthermore, western blot analyses revealed PARP cleavage in $\mathrm{TAM}^{\mathrm{R}}-4$ cells after treatment with $2 \mu \mathrm{M}$ sorafenib for $72 \mathrm{~h}$, confirming induction of apoptotic cell death (Fig. 6C).

\section{Discussion}

Resistance to endocrine therapy is a major clinical problem in the treatment of ER-positive breast cancer. Therefore, identification of drugs that target tamoxifen-resistant cells is important in order to find new treatment options. RTKs play important roles in the activation of pathways involved in cell proliferation, cell survival and ligand-independent activation of ER and may be important for circumventing the growth inhibitory effect of tamoxifen. Therefore, inhibitors of RTKs may have potential as treatment for tamoxifen-resistant breast cancer. Sorafenib and nilotinib are both multitargeting RTK inhibitors targeting a broad range of kinases important for cellular functions. We show here that both sorafenib and nilotinib preferentially inhibit the growth of tamoxifen-resistant cell lines compared with their parental tamoxifen-sensitive cell line MCF-7. Furthermore, the two inhibitors render the resistant cell lines sensitive to tamoxifen inhibition. As shown previously $(3,33,34)$, and also here, tamoxifen can stimulate growth of tamoxifen-resistant cells presumably by an agonistic effect on ER. Together these results indicate that in tamoxifen-resistant cells, sorafenib and nilotinib may switch the effect of tamoxifen from being agonistic to antagonistic, resulting in growth inhibition by tamoxifen. These results also suggest that sorafenib or nilotinib should be administered together with tamoxifen as a potential combined treatment of tamoxifen-resistant breast cancer.

Sorafenib and nilotinib are inhibitors of kinases that may signal through the MAPK or PI3K pathways. However, we did not observe inhibition but rather activation of these pathways, indicating that sorafenib and nilotinib have other mechanisms of action. Our findings that treatment with the two inhibitors lowered both the total and the phosphorylated 

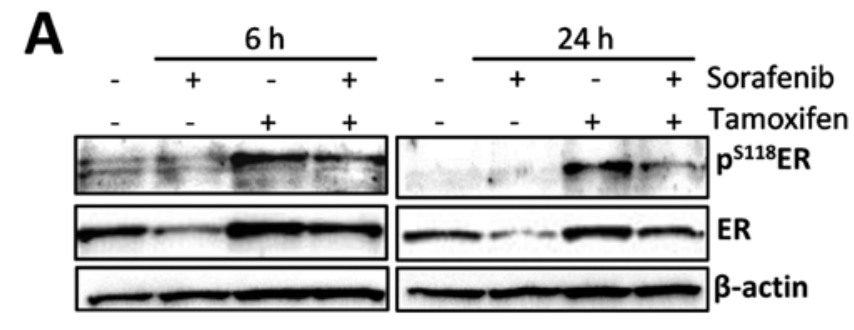

B

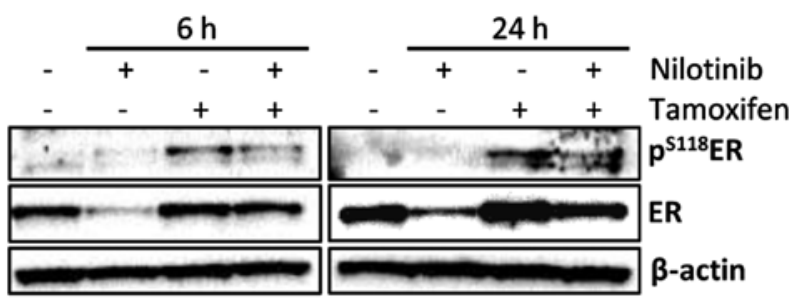

C

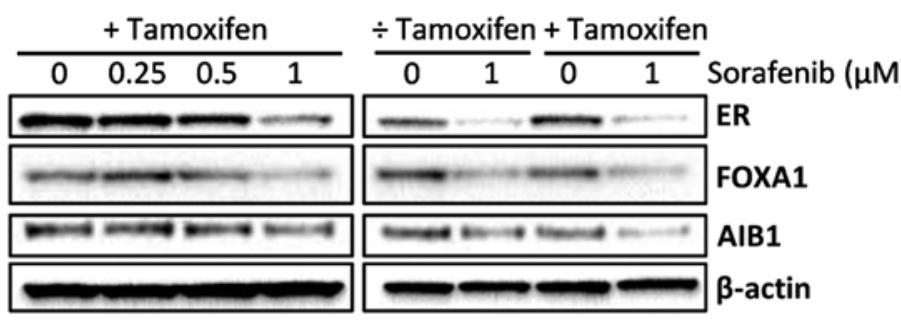

D

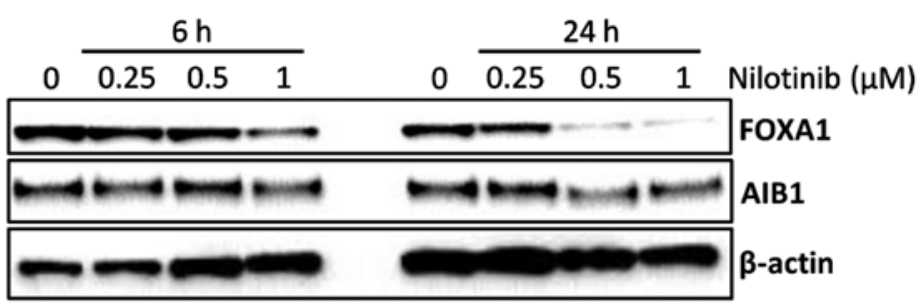

Figure 4. Effect of sorafenib and nilotinib treatment on expression of ER and its co-regulators FOXA1 and AIB1. (A and B) TAM ${ }^{\mathrm{R}}$-4 cells were withdrawn from tamoxifen one week prior to seeding and then treated for 6 or $24 \mathrm{~h}$ with $0.1 \%$ DMSO, $1 \mu \mathrm{M}$ tamoxifen, $1 \mu \mathrm{M}$ sorafenib, $1 \mu \mathrm{M}$ nilotinib or with a combination of $1 \mu \mathrm{M}$ tamoxifen and $1 \mu \mathrm{M}$ sorafenib or $1 \mu \mathrm{M}$ nilotinib as indicated before harvest for western blot analysis. (C) Tamoxifen was withdrawn from TAM ${ }^{\mathrm{R}}-4$ cells one week prior to seeding and cells were then treated with increasing concentrations of sorafenib and $1 \mu \mathrm{M}$ tamoxifen for $24 \mathrm{~h}$ or with $1 \mu \mathrm{M}$ sorafenib either with or without $1 \mu \mathrm{M}$ tamoxifen for $24 \mathrm{~h}$ before harvest. (D) TAM $\mathrm{TA}^{\mathrm{R}}-4$ cells were treated for 6 or $24 \mathrm{~h}$ with $0.1 \%$ DMSO or increasing concentrations of nilotinib as indicated before western blot analysis. $\beta$-actin was used as loading control.

level of ER and that estradiol completely abrogated the growth inhibitory effect of sorafenib and nilotinib demonstrated that the kinase inhibitors function by targeting ER activity in tamoxifen-resistant cells. Tamoxifen induces S118 phosphorylation of ER, which could contribute to the agonistic effect of tamoxifen in the resistant cells $(3,35)$. The observed lowered levels of S118-phosphorylated ER upon treatment with sorafenib or nilotinib may therefore cause growth inhibition. The tamoxifen-resistant cell lines are highly dependent on ER for growth as the ER downmodulator fulvestrant exerted severe growth inhibition of the resistant cells. Thus, the observed decrease in ER would cause growth inhibition of the tamoxifen-resistant cells. In support of our data, Weigel et al have also observed a decrease in the level of total ER and growth inhibition upon treatment of LTED cells with nilotinib (22).
We found that downregulation of ER by sorafenib and nilotinib is independent of tamoxifen, suggesting that the resensitization to tamoxifen by the inhibitors involves mechanisms, such as changes in ER co-regulators, causing a switch from agonistic to antagonistic effect of tamoxifen. Notably, we show here a decrease in the level of the two ER co-regulators AIB1 and FOXA1 upon treatment with sorafenib and nilotinib. The co-activator AIB1 is, like ER, phosphorylated by MAPKs (7) and therefore high levels of activated AIB1 could reduce the antagonistic effects of tamoxifen and cause tamoxifen resistance. In support of this, high levels of AIB1 in tumors from tamoxifen treated patients were associated with poor disease-free survival (36). Additionally, knockdown of AIB1 in the tamoxifen-resistant breast cancer cell line BT474 restored its sensitivity to tamoxifen (37). Studies have also shown that high levels of AIB1 enhance the agonistic proper- 

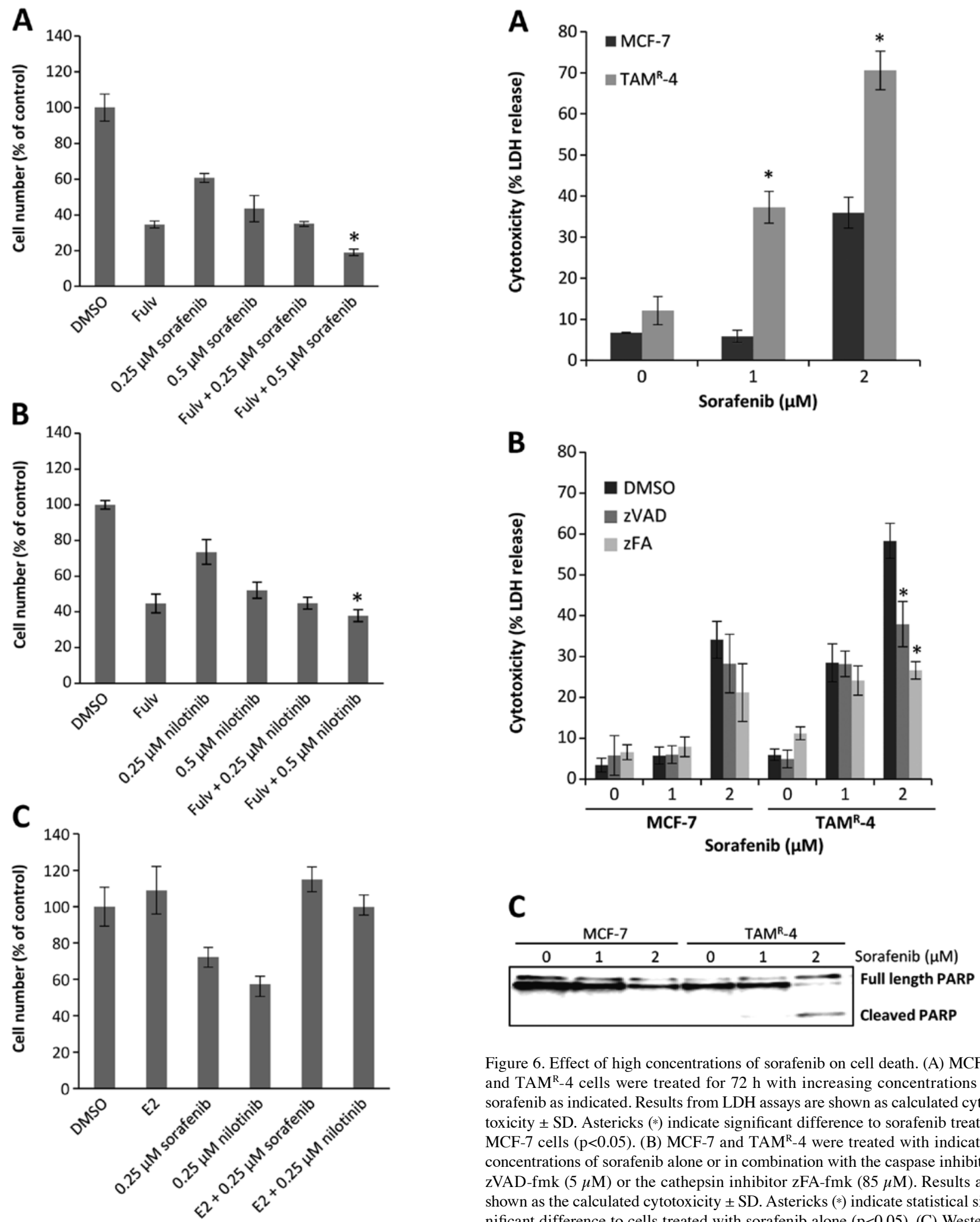

Figure 6. Effect of high concentrations of sorafenib on cell death. (A) MCF-7 and $\mathrm{TAM}^{\mathrm{R}}-4$ cells were treated for $72 \mathrm{~h}$ with increasing concentrations of sorafenib as indicated. Results from LDH assays are shown as calculated cytotoxicity \pm SD. Astericks (*) indicate significant difference to sorafenib treated MCF-7 cells $(\mathrm{p}<0.05)$. (B) MCF-7 and TAM ${ }^{\mathrm{R}}-4$ were treated with indicated concentrations of sorafenib alone or in combination with the caspase inhibitor zVAD-fmk $(5 \mu \mathrm{M})$ or the cathepsin inhibitor zFA-fmk $(85 \mu \mathrm{M})$. Results are shown as the calculated cytotoxicity \pm SD. Astericks $(*)$ indicate statistical significant difference to cells treated with sorafenib alone $(\mathrm{p}<0.05)$. (C) Western blot analysis with samples from MCF-7 and TAM ${ }^{\mathrm{R}}-4$ cells treated for $72 \mathrm{~h}$ with increasing concentrations of sorafenib as indicated.

Figure 5. Effect of sorafenib and nilotinib in combination with fulvestrant or estradiol. Tamoxifen was withdrawn from $\mathrm{TAM}^{\mathrm{R}}-4$ cells one week prior to seeding. (A) The cells were treated for 5 days with $0.1 \%$ DMSO, $10^{-7} \mathrm{M}$ fulvestrant (fulv) or sorafenib $(0.25 \mu \mathrm{M}$ and $0.5 \mu \mathrm{M})$ with and without $10^{-7} \mathrm{M}$ fulvestrant. (B) The cells were treated for 5 days with $0.1 \%$ DMSO, $10^{-7} \mathrm{M}$ fulvestrant or nilotinib $(0.25 \mu \mathrm{M}$ and $0.5 \mu \mathrm{M})$ with and without $10^{-7} \mathrm{M}$ fulvestrant. Results are expressed relative to control $(0.1 \% \mathrm{DMSO}) \pm \mathrm{SD}$. Astericks (*) indicate significant difference to DMSO or fulvestrant treated cells $(\mathrm{p}<0.05)$. (C) The cells were treated for 5 days with $0.1 \%$ DMSO, $10^{-9} \mathrm{M}$ estradiol (E2), $0.25 \mu \mathrm{M}$ sorafenib or $0.25 \mu \mathrm{M}$ nilotinib with and without $10^{-9} \mathrm{M}$ E2. Results are expressed relative to control $(0.1 \% \mathrm{DMSO}) \pm \mathrm{SD}$.

ties of tamoxifen $(38,39)$. Thus, the reduced level of AIB1 in sorafenib or nilotinib treated tamoxifen-resistant cells could cause a shift of tamoxifen from agonist to antagonist. FOXA1 is required for both the agonistic and antagonistic function of tamoxifen bound ER. Studies on a tamoxifen-resistant MCF-7-based cell line showed that silencing of FOXA1 
suppressed ligand-independent ER binding to chromatin, inhibiting cell growth (5). This demonstrates that FOXA1 is essential for ligand-independent growth of tamoxifen resistant cells. The decrease in FOXA1 protein induced by sorafenib and nilotinib may therefore reduce cell growth by inhibiting ligand-independent ER activity.

We observed that the concentration of sorafenib and nilotinib used to treat the tamoxifen-resistant cell lines was crucial for the mechanism of action of the inhibitors. At low concentration $(0.25 \mu \mathrm{M})$, only ER-dependent mechanisms were observed, while at higher concentrations $(>0.5 \mu \mathrm{M})$, a combination of both ER dependent and independent mechanisms, e.g., cytotoxic effects, were present. This is in agreement with other studies showing that sorafenib and nilotinib induce cell death at concentrations $>1 \mu \mathrm{M}$ (40-42).

In conclusion, we have shown that sorafenib and nilotinib in combination with tamoxifen inhibit growth of tamoxifen-resistant breast cancer cells. The mechanisms of action are complex and both reduced total ER and phosphorylated ER, reduced ligand-independent ER activation due to lowered FOXA1 level, and a switch in the effect of tamoxifen from agonistic to antagonistic via reduced AIB1 appears to contribute to growth inhibition. Collectively, this suggests that sorafenib or nilotinib together with tamoxifen have potential as a combined treatment of tamoxifen-resistant breast cancer patients.

\section{Acknowledgements}

We thank Jane Lind Christensen and Birgit Reiter for excellent technical assistance. This study was supported by Astrid Thaysen's grant (ATL12/01), A.P. Møller Foundation for the Advancement of Medical Science (12-374), Danish Cancer Research Foundation, Danish Cancer Society, Danske Bank Foundation, Leo Nielsen's grant (LN12/07) and Sigvald and Edith Rasmussen's grant.

\section{References}

1. Davies C, Godwin J, Gray R, et al: Relevance of breast cancer hormone receptors and other factors to the efficacy of adjuvant tamoxifen: patient-level meta-analysis of randomised trials Lancet 378: 771-784, 2011.

2. Dutertre M and Smith CL: Molecular mechanisms of selective estrogen receptor modulator (SERM) action. J Pharmacol Exp Ther 295: 431-437, 2000

3. Thrane S, Lykkesfeldt AE, Larsen MS, Sorensen BS and Yde CW: Estrogen receptor alpha is the major driving factor for growth in tamoxifen-resistant breast cancer and supported by HER/ERK signaling. Breast Cancer Res Treat 139: 71-80, 2013.

4. Lavinsky RM, Jepsen K, Heinzel T, et al: Diverse signaling pathways modulate nuclear receptor recruitment of N-CoR and SMRT complexes. Proc Natl Acad Sci USA 95: 2920-2925, 1998.

5. Hurtado A, Holmes KA, Ross-Innes CS, Schmidt D and Carroll JS: FOXA1 is a key determinant of estrogen receptor function and endocrine response. Nat Genet 43: 27-33, 2011.

6. Xu J and Li Q: Review of the in vivo functions of the p160 steroid receptor coactivator family. Mol Endocrinol 17: 1681-1692, 2003.

7. Font de Mora $J$ and Brown M: AIB1 is a conduit for kinase-mediated growth factor signaling to the estrogen receptor. Mol Cell Biol 20: 5041-5047, 2000.

8. Bunone G, Briand PA, Miksicek RJ and Picard D: Activation of the unliganded estrogen receptor by EGF involves the MAP kinase pathway and direct phosphorylation. EMBO J 15: 2174-2183, 1996

9. Kato S, Endoh H, Masuhiro Y, et al: Activation of the estrogen receptor through phosphorylation by mitogen-activated protein kinase. Science 270: 1491-1494, 1995.
10. Campbell RA, Bhat-Nakshatri P, Patel NM, Constantinidou D, Ali S and Nakshatri H: Phosphatidylinositol 3-kinase/ AKT-mediated activation of estrogen receptor alpha: a new model for anti-estrogen resistance. J Biol Chem 276: 9817-9824, 2001.

11. Zwick E, Bange J and Ullrich A: Receptor tyrosine kinase signalling as a target for cancer intervention strategies. Endocr Relat Cancer 8: 161-173, 2001.

12. Wilhelm SM, Adnane L, Newell P, Villanueva A, Llovet JM and Lynch M: Preclinical overview of sorafenib, a multikinase inhibitor that targets both Raf and VEGF and PDGF receptor tyrosine kinase signaling. Mol Cancer Ther 7: 3129-3140, 2008.

13. Plaza-Menacho I, Morandi A, Robertson D, et al: Targeting the receptor tyrosine kinase RET sensitizes breast cancer cells to tamoxifen treatment and reveals a role for RET in endocrine resistance. Oncogene 29: 4648-4657, 2010.

14. Escudier B, Eisen T, Stadler WM, et al: Sorafenib in advanced clear-cell renal-cell carcinoma. N Engl J Med 356: 125-134, 2007.

15. Llovet JM, Ricci S, Mazzaferro V, et al: Sorafenib in advanced hepatocellular carcinoma. N Engl J Med 359: 378-390, 2008.

16. Luu T, Frankel P, Chung C, et al: Phase I/II trial of vinorelbine and sorafenib in metastatic breast cancer. Clin Breast Cancer 14: 94-100, 2014.

17. Baselga J, Costa F, Gomez H, et al: A phase 3 trial comparing capecitabine in combination with sorafenib or placebo for treatment of locally advanced or metastatic HER2-negative breast cancer (the RESILIENCE study): study protocol for a randomized controlled trial. Trials 14: 228, 2013

18. Wilhelm SM, Carter C, Tang L, et al: BAY 43-9006 exhibits broad spectrum oral antitumor activity and targets the $\mathrm{RAF} / \mathrm{MEK} / \mathrm{ERK}$ pathway and receptor tyrosine kinases involved in tumor progression and angiogenesis. Cancer Res 64: 7099-7109, 2004.

19. Weisberg E, Manley P, Mestan J, Cowan-Jacob S, Ray A and Griffin JD: AMN107 (nilotinib): a novel and selective inhibitor of BCR-ABL. Br J Cancer 94: 1765-1769, 2006.

20. O'Hare T, Walters DK, Deininger MW and Druker BJ: AMN107: tightening the grip of imatinib. Cancer Cell 7: 117-119, 2005.

21. Piccaluga PP, Paolini S, Bertuzzi C, De Leo A and Rosti G: First-line treatment of chronic myeloid leukemia with nilotinib: critical evaluation. J Blood Med 3: 151-156, 2012.

22. Weigel MT, Ghazoui Z, Dunbier A, Pancholi S, Dowsett M and Martin LA: Preclinical and clinical studies of estrogen deprivation support the PDGF/Abl pathway as a novel therapeutic target for overcoming endocrine resistance in breast cancer. Breast Cancer Res 14: R78, 2012.

23. Briand P and Lykkesfeldt AE: Effect of estrogen and antiestrogen on the human breast cancer cell line MCF-7 adapted to growth at low serum concentration. Cancer Res 44: 1114-1119, 1984.

24. Lykkesfeldt AE, Madsen MW and Briand P: Altered expression of estrogen-regulated genes in a tamoxifen-resistant and ICI 164,384 and ICI 182,780 sensitive human breast cancer cell line, MCF-7/TAM ${ }^{\mathrm{R}}-1$. Cancer Res 54: 1587-1595, 1994.

25. Lundholt BK, Briand P and Lykkesfeldt AE: Growth inhibition and growth stimulation by estradiol of estrogen receptor transfected human breast epithelial cell lines involve different pathways. Breast Cancer Res Treat 67: 199-214, 2001.

26. Larsen MS, Yde CW, Christensen IJ and Lykkesfeldt AE: Carboplatin treatment of antiestrogen-resistant breast cancer cells. Int J Oncol 41: 1863-1870, 2012.

27. Arnold SF, Obourn JD, Jaffe H and Notides AC: Phosphorylation of the human estrogen receptor by mitogen-activated protein kinase and casein kinase II: consequence on DNA binding. J Steroid Biochem Mol Biol 55: 163-172, 1995.

28. De Leeuw R, Neefjes J and Michalides R: A role for estrogen receptor phosphorylation in the resistance to tamoxifen. Int $\mathrm{J}$ Breast Cancer 2011: 232435, 2011.

29. Chen D, Riedl T, Washbrook E, et al: Activation of estrogen receptor alpha by S118 phosphorylation involves a liganddependent interaction with TFIIH and participation of CDK7. Mol Cell 6: 127-137, 2000.

30. Wijayaratne AL and McDonnell DP: The human estrogen receptor-alpha is a ubiquitinated protein whose stability is affected differentially by agonists, antagonists, and selective estrogen receptor modulators. J Biol Chem 276: 35684-35692, 2001.

31. Osborne CK, Wakeling A and Nicholson RI: Fulvestrant: an oestrogen receptor antagonist with a novel mechanism of action. Br J Cancer 90 (Suppl 1): S2-S6, 2004. 
32. Kroemer G and Jaattela M: Lysosomes and autophagy in cell death control. Nat Rev Cancer 5: 886-897, 2005.

33. Shou J, Massarweh S, Osborne CK, et al: Mechanisms of tamoxifen resistance: increased estrogen receptor-HER2/neu cross-talk in ER/HER2-positive breast cancer. J Natl Cancer Inst 96: 926-935, 2004.

34. Hodges LC, Cook JD, Lobenhofer EK, et al: Tamoxifen functions as a molecular agonist inducing cell cycle-associated genes in breast cancer cells. Mol Cancer Res 1: 300-311, 2003.

35. Vendrell JA, Bieche I, Desmetz C, et al: Molecular changes associated with the agonist activity of hydroxy-tamoxifen and the hyper-response to estradiol in hydroxy-tamoxifen-resistant breast cancer cell lines. Endocr Relat Cancer 12: 75-92, 2005.

36. Osborne CK, Bardou V, Hopp TA, et al: Role of the estrogen receptor coactivator AIB1 (SRC-3) and HER-2/neu in tamoxifen resistance in breast cancer. J Natl Cancer Inst 95: 353-361, 2003.

37. Su Q, Hu S, Gao H, et al: Role of AIB1 for tamoxifen resistance in estrogen receptor-positive breast cancer cells. Oncology 75 : $159-168,2008$

38. Smith CL, Nawaz Z and O'Malley BW: Coactivator and corepressor regulation of the agonist/antagonist activity of the mixed antiestrogen, 4-hydroxytamoxifen. Mol Endocrinol 11: 657-666, 1997.
39. Reiter R, Oh AS, Wellstein A and Riegel AT: Impact of the nuclear receptor coactivator AIB1 isoform AIB1-Delta3 on estrogenic ligands with different intrinsic activity. Oncogene 23 : 403-409, 2004

40. Rahmani M, Davis EM, Crabtree TR, et al: The kinase inhibitor sorafenib induces cell death through a process involving induction of endoplasmic reticulum stress. Mol Cell Biol 27: 5499-5513, 2007.

41. LiuL,Cao Y,Chen C, et al: Sorafenib blocks the RAF/MEK/ERK pathway, inhibits tumor angiogenesis, and induces tumor cell apoptosis in hepatocellular carcinoma model PLC/PRF/5. Cancer Res 66: 11851-11858, 2006.

42. Shaker ME, Ghani A, Shiha GE, Ibrahim TM and Mehal WZ: Nilotinib induces apoptosis and autophagic cell death of activated hepatic stellate cells via inhibition of histone deacetylases. Biochim Biophys Acta 1833: 1992-2003, 2013. 